\title{
General $L_{p}$-mixed width-integral of convex bodies and related inequalities
}

\section{Yanping Zhou}

Department of Mathematics, Shanghai University, Shanghai 200444, China.

\begin{abstract}
The conception of general $\mathrm{L}_{\mathrm{p}}$-mixed width-integral of convex bodies is introduced and related isoperimetric type inequality, Aleksandrov-Fenchel type inequality and a cyclic inequality are established. Further, the extremum values for the general $\mathrm{L}_{\mathrm{p}}{ }^{-}$ mixed width-integral are obtained. (c)2017 All rights reserved.
\end{abstract}

Keywords: General $\mathrm{L}_{\mathrm{p}}$-mixed width-integral, general mixed width-integral, mixed width-integral, convex body. 2010 MSC: 52A20, 52A40.

\section{Introduction and main results}

Let $\mathcal{K}^{n}$ denote the set of convex bodies (compact, convex subsets with non-empty interiors) in Euclidean space $\mathbb{R}^{n}$. For the set of convex bodies containing the origin in their interiors and the set of convex bodies whose centroids lie at the origin in $\mathbb{R}^{n}$, we write $\mathcal{K}_{0}^{n}$ and $\mathcal{K}_{c}^{n}$, respectively. Let $S^{n-1}$ denote the unit sphere in $\mathbb{R}^{n}$, and let $V(K)$ denote the $n$-dimensional volume of a body $K$. For the standard unit ball $B$ in $\mathbb{R}^{n}$, we use $\omega_{n}=V(B)$ to denote its volume.

If $K \in \mathcal{K}^{n}$, then its support function, $h_{K}=h(K, \cdot): \mathbb{R}^{n} \rightarrow(-\infty, \infty)$, is defined by $($ see $[9,30])$

$$
h(K, x)=\max \{x \cdot y: y \in K\}, x \in \mathbb{R}^{n},
$$

where $x \cdot y$ denotes the standard inner product of $x$ and $y$.

The study of width integral has a long history. The notion of the classical width-integral was first considered by Blaschke (see [4]) and was further studied by Hardy et al. (see [16]). It was generalized to the mixed width-integral by Lutwak [23] in 1977. For the more results associated with the mixed widthintegral, we refer the interested reader to [17, 21, 22, 25]. Particularly, Feng [6] generalized the definition of mixed width integral to general mixed width integral: for $\tau \in(-1,1)$, the general mixed width-integral, $\mathrm{B}^{(\tau)}\left(\mathrm{K}_{1}, \cdots, \mathrm{K}_{n}\right)$, of $\mathrm{K}_{1}, \cdots, \mathrm{K}_{\mathrm{n}} \in \mathcal{K}_{\mathrm{o}}^{\mathrm{n}}$ is defined by

$$
B^{(\tau)}\left(K_{1}, \cdots, K_{n}\right)=\frac{1}{n} \int_{S^{n-1}} b^{(\tau)}\left(K_{1}, u\right) \cdots b^{(\tau)}\left(K_{n}, u\right) d S(u),
$$

Email address: zhouyp@i.shu.edu.cn (Yanping Zhou)

doi:10.22436/jnsa.010.08.30 
where $b^{(\tau)}(K, u)=f_{1}(\tau) h(K, u)+f_{2}(\tau) h(K,-u)$ and the functions $f_{1}(\tau)$ and $f_{2}(\tau)$ are chosen as follows

$$
f_{1}(\tau)=\frac{(1+\tau)^{2}}{2\left(1+\tau^{2}\right)}, \quad f_{2}(\tau)=\frac{(1-\tau)^{2}}{2\left(1+\tau^{2}\right)} .
$$

The main aim of this article is to define a corresponding notion of general $L_{p}$-mixed width-integral, and to extend Feng's inequalities to the inequalities related to general $\mathrm{L}_{\mathrm{p}}$-mixed width-integral. Moreover, the extremum values and two Brunn-Minkowski type inequalities for the general $L_{p}$-mixed width-integral are established.

Now, we define the general $L_{p}$-mixed width-integral as follows: for $\tau \in[-1,1]$ and $p>0$, the general $\mathrm{L}_{\mathrm{p}}$-mixed width-integral, $\mathrm{B}_{\mathrm{p}}^{(\tau)}\left(\mathrm{K}_{1}, \cdots, \mathrm{K}_{n}\right)$, of $\mathrm{K}_{1}, \cdots, \mathrm{K}_{n} \in \mathcal{K}_{\mathrm{o}}^{n}$ is defined by

$$
B_{p}^{(\tau)}\left(K_{1}, \cdots, K_{n}\right)=\frac{1}{n} \int_{S^{n-1}} b_{p}^{(\tau)}\left(K_{1}, u\right) \cdots b_{p}^{(\tau)}\left(K_{n}, u\right) d S(u),
$$

where $b_{p}^{(\tau)}(K, u)=\left(f_{1}(\tau) h^{p}(K, u)+f_{2}(\tau) h^{p}(K,-u)\right)^{\frac{1}{p}}$ for any $u \in S^{n-1}$, and $f_{1}(\tau), f_{2}(\tau)$ are chosen as follows

$$
f_{1}(\tau)=\frac{(1+\tau)^{2 p}}{(1+\tau)^{2 p}+(1-\tau)^{2 p}}, \quad f_{2}(\tau)=\frac{(1-\tau)^{2 p}}{(1+\tau)^{2 p}+(1-\tau)^{2 p}}
$$

Obviously, $f_{1}(\tau)$ and $f_{2}(\tau)$ satisfy

$$
\begin{aligned}
f_{1}(\tau)+f_{2}(\tau) & =1, \\
f_{1}(-\tau) & =f_{2}(\tau), \quad f_{2}(-\tau)=f_{1}(\tau) .
\end{aligned}
$$

Note that the case $p=1$ in definition (1.1) is just Feng's general mixed width-integral. Two convex bodies $\mathrm{K}$ and $\mathrm{L}$ are said to have similar general $\mathrm{L}_{\mathrm{p}}$-width if there exists a constant $\lambda>0$ such that $b_{p}^{(\tau)}(K, u)=\lambda b_{p}^{(\tau)}(L, u)$ for all $u \in S^{n-1}$. If $b_{p}^{(\tau)}(K, u)=b_{p}^{(\tau)}(L, u)$ for all $u \in S^{n-1}$, then we call $K$ and $L$ have the same general $\mathrm{L}_{p}$-width.

Taking $\mathrm{K}_{1}=\cdots=\mathrm{K}_{\mathrm{n}-\mathrm{i}}=\mathrm{K}$ and $\mathrm{K}_{\mathrm{n}-\mathrm{i}+1}=\cdots=\mathrm{K}_{\mathrm{n}}=\mathrm{L}$ in (1.1), the general $\mathrm{L}_{\mathrm{p}}$-mixed width-integral, $\mathrm{B}_{\mathrm{p}, \mathrm{i}}^{(\tau)}(\mathrm{K}, \mathrm{L})$, of $\mathrm{K}, \mathrm{L} \in \mathcal{K}_{\mathrm{o}}^{\mathrm{n}}$ is given by

$$
B_{p, i}^{(\tau)}(K, L)=\frac{1}{n} \int_{S^{n-1}} b_{p}^{(\tau)}(K, u)^{n-i} b_{p}^{(\tau)}(L, u)^{i} d S(u)
$$

Further, let $L=B$ in (1.2). Then we write $B_{p, i}^{(\tau)}(K)$ for $B_{p, i}^{(\tau)}(K, B)$. Since $b_{p}^{(\tau)}(B, u)=1$, it follows that

$$
\mathrm{B}_{p, i}^{(\tau)}(K)=\frac{1}{n} \int_{S^{n-1}} b_{p}^{(\tau)}(K, u)^{n-i} d S(u)
$$

where $B_{p, i}^{(\tau)}(K)$ is called the ith general $L_{p}$-mixed width-integral of $K$. If $i=0$, then we write $B_{p, 0}^{(\tau)}(K)=$ $\mathrm{B}_{\mathrm{p}}^{(\tau)}(\mathrm{K})$, which we call the general $\mathrm{L}_{\mathrm{p}}$-width-integral of $K$, namely,

$$
\mathrm{B}_{\mathrm{p}}^{(\tau)}(\mathrm{K})=\frac{1}{\mathrm{n}} \int_{\mathrm{S}^{\mathrm{n}-1}} \mathrm{~b}_{\mathrm{p}}^{(\tau)}(\mathrm{K}, \mathrm{u})^{\mathrm{n}} \mathrm{dS}(\mathrm{u})
$$

The general operator belongs to the asymmetric Brunn-Minkowski theory which has its starting point in the theory of valuations in connection with isoperimetric and analytic inequalities (see $[1,2,7,8,10$ $15,19,20,27-29,31-35])$.

The main results are the following: we first establish the isoperimetric type, Aleksandrov-Fenchel type and cyclic inequalities for the general $\mathrm{L}_{p}$-mixed width-integral.

Theorem 1.1. If $\tau \in[-1,1]$ and $\mathrm{K}_{1}, \cdots, \mathrm{K}_{\mathrm{n}} \in \mathcal{K}_{\mathrm{c}}^{\mathrm{n}}$, then for $\mathrm{p}>0$

$$
\mathrm{V}\left(\mathrm{K}_{1}\right) \cdots \mathrm{V}\left(\mathrm{K}_{\mathrm{n}}\right) \leqslant \mathrm{B}_{\mathrm{p}}^{(\tau)}\left(\mathrm{K}_{1}, \cdots, \mathrm{K}_{\mathrm{n}}\right)^{\mathrm{n}},
$$

with equality if and only if $\mathrm{K}_{1}, \cdots, \mathrm{K}_{\mathrm{n}}$ are $n$-balls. 
Theorem 1.2. If $\tau \in[-1,1], \mathrm{K}_{1}, \cdots, \mathrm{K}_{\mathrm{n}} \in \mathcal{K}_{\mathrm{o}}^{\mathrm{n}}$, and $1<\mathrm{m} \leqslant \mathrm{n}$, then for $\mathrm{p}>0$

$$
B_{p}^{(\tau)}\left(K_{1}, \cdots, K_{n}\right)^{m} \leqslant \prod_{i=1}^{m} B_{p}^{(\tau)}\left(K_{1}, \cdots, K_{n-m}, K_{n-i+1}, \cdots, K_{n-i+1}\right),
$$

with equality if and only if $\mathrm{K}_{\mathrm{n}-\mathrm{m}+1}, \cdots, \mathrm{K}_{\mathrm{n}}$ are all of similar general $\mathrm{L}_{\mathrm{p}}$-width.

Theorem 1.3. If $\tau \in[-1,1], K, L \in \mathcal{K}_{0}^{n}$, and $p>0$, then for $i<j<k$,

$$
\mathrm{B}_{\mathrm{p}, \mathrm{i}}^{(\tau)}(\mathrm{K}, \mathrm{L})^{\mathrm{k}-\mathrm{j}} \mathrm{B}_{\mathrm{p}, \mathrm{k}}^{(\tau)}(\mathrm{K}, \mathrm{L})^{\mathrm{j}-\mathrm{i}} \geqslant \mathrm{B}_{\mathrm{p}, \mathrm{j}}^{(\tau)}(\mathrm{K}, \mathrm{L})^{\mathrm{k}-\mathrm{i}},
$$

with equality if and only if $\mathrm{K}$ and $\mathrm{L}$ have similar general $\mathrm{L}_{\mathrm{p}}$-width.

Moreover, the extremum values for the general $\mathrm{L}_{\mathrm{p}}$-mixed width-integral are also established.

Theorem 1.4. If $\tau \in[-1,1], K \in \mathcal{K}_{0}^{n}$, and $p>0$, then for $i<n-p$,

$$
\mathrm{B}_{p, i}^{(0)}(\mathrm{K}) \leqslant \mathrm{B}_{p, i}^{(\tau)}(\mathrm{K}) \leqslant \mathrm{B}_{p, i}^{( \pm 1)}(\mathrm{K})
$$

for $n-p<i<n$ or $i>n$

$$
\mathrm{B}_{p, i}^{(0)}(\mathrm{K}) \geqslant \mathrm{B}_{\mathrm{p}, \mathrm{i}}^{(\tau)}(\mathrm{K}) \geqslant \mathrm{B}_{\mathrm{p}, \mathrm{i}}^{( \pm 1)}(\mathrm{K})
$$

If $\mathrm{K}$ is not origin-symmetric, equality holds in the left inequality if and only if $\tau=0$ and equality holds in the right inequality if and only if $\tau= \pm 1$. When $i=n,(1.7)$ and (1.8) are identical.

The proofs of theorems above will be given in Section 3 of this paper. In Section 4, two BrunnMinkowski type inequalities for the ith general $\mathrm{L}_{p}$-mixed width-integral will be established.

\section{Preliminaries}

The radial function, $\rho_{K}=\rho(K, \cdot): \mathbb{R}^{n} \backslash\{0\} \rightarrow[0, \infty)$, of a compact star-shaped (about the origin) set $K$ in $\mathbb{R}^{n}$ is defined, for $u \in S^{n-1}$, by (see $\left.[9,30]\right)$

$$
\rho(K, u)=\max \{\lambda \geqslant 0: \lambda \cdot u \in K\} .
$$

The polar body, $\mathrm{K}^{*}$, of $\mathrm{K} \in \mathcal{K}^{\mathrm{n}}$ is defined by (see $\left.[9,30]\right)$

$$
K^{*}=\left\{x \in \mathbb{R}^{n}: x \cdot y \leqslant 1, y \in K\right\} .
$$

It is easy to check that for $\mathrm{K} \in \mathcal{K}_{\mathrm{o}}^{\mathrm{n}}$,

$$
\left(\mathrm{K}^{*}\right)^{*}=\mathrm{K} \quad \text { and } \quad \mathrm{h}_{\mathrm{K}^{*}}=\frac{1}{\rho_{\mathrm{K}}}, \rho_{\mathrm{K}^{*}}=\frac{1}{\mathrm{~h}_{\mathrm{K}}} .
$$

An extension of the well-known Blaschke-Santaló inequality is as follows (see [24]).

Theorem 2.1. If $\mathrm{K} \in \mathcal{K}_{\mathrm{c}}^{\mathrm{n}}$, then

$$
\mathrm{V}(\mathrm{K}) \mathrm{V}\left(\mathrm{K}^{*}\right) \leqslant \omega_{\mathrm{n}}^{2}
$$

with equality if and only if $\mathrm{K}$ is an ellipsoid.

For $K, L \in \mathcal{K}_{\mathrm{o}}^{n}, \mathrm{p} \geqslant 1$ and $\lambda, \mu \geqslant 0$ (not both zero), the Firey $\mathrm{L}_{\mathrm{p}}$-combination (also called $\mathrm{L}_{\mathrm{p}}$-Minkowski combination), $\lambda \cdot K+_{p} \mu \cdot L \in \mathcal{K}_{o}^{n}$, of $K$ and $L$ is defined by (see [26])

$$
h\left(\lambda \cdot K+_{p} \mu \cdot L_{,} \cdot\right)^{p}=\lambda h(K, \cdot)^{p}+\mu h(L, \cdot)^{p},
$$

where the operation " $+p$ " is called Firey addition and $\lambda \cdot K$ denotes the Firey scalar multiplication.

The polar coordinate formula for volume of a body $K$ in $\mathbb{R}^{n}$ is

$$
V(K)=\frac{1}{n} \int_{S^{n-1}} \rho(K, u)^{n} d S(u)
$$




\section{Proof of main results}

Proof of Theorem 1.1. From Jensen's inequality (see [16]), we obtain

$$
\begin{aligned}
B_{p}^{(\tau)}\left(K_{1}, \cdots, K_{n}\right) & =\frac{1}{n} \int_{S^{n-1}} b_{p}^{(\tau)}\left(K_{1}, u\right) \cdots b_{p}^{(\tau)}\left(K_{n}, u\right) d S(u) \\
& \geqslant n \omega_{n}^{2}\left[\int_{S^{n-1}} b_{p}^{(\tau)}\left(K_{1}, u\right)^{-1} \cdots b_{p}^{(\tau)}\left(K_{n}, u\right)^{-1} d S(u)\right]^{-1}
\end{aligned}
$$

with equality if and only if $\mathrm{K}_{1}, \cdots, \mathrm{K}_{\mathrm{n}}$ have joint constant general $\mathrm{L}_{\mathrm{p}}$-width. Together with Hölder's inequality (see [16]), we have

$$
\left[\int_{S^{n-1}} b_{p}^{(\tau)}\left(K_{1}, u\right)^{-1} \cdots b_{p}^{(\tau)}\left(K_{n}, u\right)^{-1} d S(u)\right]^{-n} \geqslant \prod_{i=1}^{n}\left[\int_{S^{n-1}} b_{p}^{(\tau)}\left(K_{i}, u\right)^{-n} d S(u)\right]^{-1},
$$

with equality if and only if $\mathrm{K}_{1}, \cdots, \mathrm{K}_{\mathrm{n}}$ have similar general $\mathrm{L}_{\mathrm{p}}$-width. Using Minkowski's inequality (see [16]), we have

$$
\begin{aligned}
{\left[\frac{1}{n} \int_{S^{n-1}} b_{p}^{(\tau)}\left(K_{i}, u\right)^{-n} d S(u)\right]^{-\frac{p}{n}}=} & {\left[\frac{1}{n} \int_{S^{n-1}}\left(f_{1}(\tau) h^{p}\left(K_{i}, u\right)+f_{2}(\tau) h^{p}\left(K_{i},-u\right)\right)^{-\frac{n}{p}} d S(u)\right]^{-\frac{p}{n}} } \\
\geqslant & {\left[\frac{1}{n} \int_{S^{n-1}}\left(f_{1}(\tau) h^{p}\left(K_{i}, u\right)\right)^{-\frac{n}{p}} d S(u)\right]^{-\frac{p}{n}} } \\
& +\left[\frac{1}{n} \int_{S^{n-1}}\left(f_{2}(\tau) h^{p}\left(K_{i},-u\right)\right)^{-\frac{n}{p}} d S(u)\right]^{-\frac{p}{n}} \\
\geqslant & {\left[\frac{1}{n} \int_{S^{n-1}} h\left(K_{i}, u\right)^{-n} d S(u)\right]^{-\frac{p}{n}}=V\left(K_{i}^{*}\right)^{-\frac{p}{n}} }
\end{aligned}
$$

with equality if and only if $K_{i}$ is origin-symmetric. Since $p>0$, it follows from Theorem 2.1 and inequality (3.3) that,

$$
\left[\frac{1}{n \omega_{n}^{2}} \int_{S^{n-1}} b_{p}^{(\tau)}\left(K_{i}, u\right)^{-n} d S(u)\right]^{-1} \geqslant V\left(K_{i}\right),
$$

with equality if and only if $K_{i}$ is an n-dimensional ellipsoid. By inequalities (3.1), (3.2), and (3.4), this gives

$$
V\left(K_{1}\right) \cdots V\left(K_{n}\right) \leqslant B_{p}^{(\tau)}\left(K_{1}, \cdots, K_{n}\right)^{n}
$$

By the equality conditions of inequalities (3.1), (3.2), and (3.4), equality holds in (1.4) if and only if $\mathrm{K}_{1}, \cdots, \mathrm{K}_{\mathrm{n}}$ are $\mathrm{n}$-balls.

Lemma 3.1 ([22]). If $\mathrm{f}_{0}, \mathrm{f}_{1}, \cdots, \mathrm{f}_{\mathrm{m}}$ are (strictly) positive continuous functions defined on $\mathrm{S}^{\mathrm{n}-1}$ and $\lambda_{1}, \cdots, \lambda_{\mathrm{m}}$ are positive constants the sum of whose reciprocals is unity, then

$$
\int_{S^{n-1}} f_{0}(u) f_{1}(u) \cdots f_{m}(u) d S(u) \leqslant \prod_{i=1}^{m}\left[\int_{S^{n-1}} f_{0}(u) f_{i}^{\lambda_{i}}(u) d S(u)\right]^{\frac{1}{\lambda_{i}}}
$$

with equality if and only if there exist positive constants $\alpha_{1}, \cdots, \alpha_{m}$ such that $\alpha_{1} f_{1}^{\lambda_{1}}(u)=\cdots=\alpha_{m} f_{m}^{\lambda_{m}}(u)$ for all $u \in \mathrm{S}^{\mathrm{n}-1}$.

Proof of Theorem 1.2. Let in Lemma 3.1

$$
\begin{aligned}
& \lambda_{i}=m \quad(1 \leqslant i \leqslant m) ; \\
& f_{0}=b_{p}^{(\tau)}\left(K_{1}, u\right) \cdots b_{p}^{(\tau)}\left(K_{n-m}, u\right) \quad\left(f_{0}=1 \text { if } m=n\right) ;
\end{aligned}
$$




$$
f_{i}=b_{p}^{(\tau)}\left(K_{n-i+1}, u\right) \quad(1 \leqslant i \leqslant m) .
$$

Then

$$
\begin{aligned}
& \int_{S^{n-1}} b_{p}^{(\tau)}\left(K_{1}, u\right) \cdots b_{p}^{(\tau)}\left(K_{n}, u\right) d S(u) \\
& \leqslant \prod_{i=1}^{m}\left[\int_{S^{n-1}} b_{p}^{(\tau)}\left(K_{1}, u\right) \cdots b_{p}^{(\tau)}\left(K_{n-m}, u\right) b_{p}^{(\tau)}\left(K_{n-i+1}, u\right)^{m} d S(u)\right]^{\frac{1}{m}} .
\end{aligned}
$$

Combining with (1.1), we have

$$
B_{p}^{(\tau)}\left(K_{1}, \cdots, K_{n}\right)^{m} \leqslant \prod_{i=1}^{m} B_{p}^{(\tau)}\left(K_{1}, \cdots, K_{n-m}, K_{n-i+1}, \cdots, K_{n-i+1}\right) .
$$

The equality condition of inequality (3.5) implies that equality holds in (1.5) if and only if $K_{n-m+1}$, $\cdots, \mathrm{K}_{\mathrm{n}}$ are all of similar general $\mathrm{L}_{\mathrm{p}}$-width.

Proof of Theorem 1.3. It follows from Hölder's inequality (see [16]) that

$$
\begin{aligned}
& B_{p, i}^{(\tau)}(K, L)^{\frac{k-j}{k-i}} B_{p, k}^{(\tau)}(K, L)^{\frac{j-i}{k-i}}=\left(\frac{1}{n} \int_{S^{n-1}} b_{p}^{(\tau)}(K, u)^{n-i} b_{p}^{(\tau)}(L, u)^{i} d S(u)\right)^{\frac{k-j}{k-i}} \\
& \times\left(\frac{1}{n} \int_{S^{n-1}} b_{p}^{(\tau)}(K, u)^{n-k} b_{p}^{(\tau)}(L, u)^{k} d S(u)\right)^{\frac{j-i}{k-i}} \\
& \geqslant \frac{1}{n} \int_{S^{n-1}} b_{p}^{(\tau)}(K, u)^{n-j} b_{p}^{(\tau)}(L, u)^{j} d S(u)=B_{p, j}^{(\tau)}(K, L) .
\end{aligned}
$$

This gives

$$
B_{p, i}^{(\tau)}(K, L)^{k-j} B_{p, k}^{(\tau)}(K, L)^{j-i} \geqslant B_{p, j}^{(\tau)}(K, L)^{k-i}
$$

The equality condition of Hölder's inequality implies that equality holds in (1.6) if and only if K and L have similar general $\mathrm{L}_{\mathrm{p}}$-width.

Taking $i=0, j=i$, and $k=n$ in inequality (1.6), we have the following.

Corollary 3.2. If $\tau \in[-1,1], K, L \in \mathcal{K}_{0}^{n}$, and $p>0$, then for $0 \leqslant i \leqslant n$,

$$
\mathrm{B}_{\mathrm{p}, \mathrm{i}}^{(\tau)}(\mathrm{K}, \mathrm{L})^{\mathrm{n}} \leqslant \mathrm{B}_{\mathrm{p}}^{(\tau)}(\mathrm{K})^{\mathrm{n}-\mathrm{i}} \mathrm{B}_{\mathrm{p}}^{(\tau)}(\mathrm{L})^{\mathrm{i}}
$$

for $i<0$ or $i>n$, inequality (3.6) is reversed, with equality in every inequality if and only if $i=n$ or, when $i \neq n$, $\mathrm{K}$ and $\mathrm{L}$ have similar general $\mathrm{L}_{\mathrm{p}}$-width.

Let $i=1$ in Corollary 3.2. The dual Minkowski type inequalities for the general $L_{p}$-mixed widthintegral are as follows.

Corollary 3.3. If $\tau \in[-1,1]$ and $\mathrm{K}, \mathrm{L} \in \mathcal{K}_{\mathrm{o}}^{\mathrm{n}}$, then

$$
\mathrm{B}_{\mathrm{p}, 1}^{(\tau)}(\mathrm{K}, \mathrm{L})^{\mathrm{n}} \leqslant \mathrm{B}_{\mathrm{p}}^{(\tau)}(\mathrm{K})^{\mathrm{n}-1} \mathrm{~B}_{\mathrm{p}}^{(\tau)}(\mathrm{L})
$$

with equality if and only if $\mathrm{K}$ and $\mathrm{L}$ have similar general $\mathrm{L}_{\mathrm{p}}$-width.

Corollary 3.4. If $\tau \in[-1,1]$ and $\mathrm{K}, \mathrm{L} \in \mathcal{K}_{\mathrm{o}}^{\mathrm{n}}$, then

$$
\mathrm{B}_{\mathrm{p},-1}^{(\tau)}(\mathrm{K}, \mathrm{L})^{\mathrm{n}} \geqslant \mathrm{B}_{\mathrm{p}}^{(\tau)}(\mathrm{K})^{\mathrm{n}+1} \mathrm{~B}_{\mathrm{p}}^{(\tau)}(\mathrm{L})^{-1},
$$

with equality if and only if $\mathrm{K}$ and $\mathrm{L}$ have similar general $\mathrm{L}_{\mathrm{p}}$-width. 
Proof of Theorem 1.4. From (1.3) and using Minkowski's inequality (see [16]), we obtain that for $i<n-p$

$$
\begin{aligned}
B_{p, i}^{(\tau)}(K) \frac{p}{n-i}= & {\left[\frac{1}{n} \int_{S^{n-1}} b_{p}^{(\tau)}(K, u)^{n-i} d S(u)\right]^{\frac{p}{n-i}} } \\
= & {\left[\frac{1}{n} \int_{S^{n-1}}\left(f_{1}(\tau) h^{p}(K, u)+f_{2}(\tau) h^{p}(K,-u)\right)^{\frac{n-i}{p}} d S(u)\right]^{\frac{p}{n-i}} } \\
\leqslant & {\left[\frac{1}{n} \int_{S^{n-1}}\left(f_{1}(\tau) h^{p}(K, u)\right)^{\frac{n-i}{p}} d S(u)\right]^{\frac{p}{n-i}} } \\
& +\left[\frac{1}{n} \int_{S^{n-1}}\left(f_{2}(\tau) h^{p}(K,-u)\right)^{\frac{n-i}{p}} d S(u)\right]^{\frac{p}{n-i}} \\
= & {\left[\frac{1}{n} \int_{S^{n-1}}\left(h^{p}(K, u)\right)^{\frac{n-i}{p}} d S(u)\right]^{\frac{p}{n-i}} \text { or }\left[\frac{1}{n} \int_{S^{n-1}}\left(h^{p}(K,-u)\right)^{\frac{n-i}{p}} d S(u)\right]^{\frac{p}{n-i}} } \\
= & {\left[\frac{1}{n} \int_{S^{n-1}}(h(K, u))^{n-i} d S(u)\right]^{\frac{p}{n-i}} \cdot }
\end{aligned}
$$

Similarly, from Minkowski's inequality we have that for $n-p<i<n$ or $i<n$,

$$
B_{p, i}^{(\tau)}(K)^{\frac{p}{n-i}} \geqslant\left[\frac{1}{n} \int_{S^{n-1}}(h(K, u))^{n-i} d S(u)\right]^{\frac{p}{n-i}} .
$$

From the equality condition of Minkowski's inequality, we see that equalities hold in (3.6) and (3.7) if and only if $\mathrm{K}$ and $-\mathrm{K}$ are dilates of one another, i.e., $\mathrm{K}$ is centered.

From $f_{1}(+1)=1$ and $f_{2}(+1)=0$, we have

$$
B_{p, i}^{(+1)}(K)=\frac{1}{n} \int_{S^{n-1}}\left(h^{p}(K, u)\right)^{\frac{n-i}{p}} d S(u)=\frac{1}{n} \int_{S^{n-1}}(h(K, u))^{n-i} d S(u) .
$$

Together with (3.7), we have

$$
\mathrm{B}_{\mathrm{p}, \mathrm{i}}^{(\tau)}(\mathrm{K}) \leqslant \mathrm{B}_{\mathrm{p}, \mathrm{i}}^{( \pm 1)}(\mathrm{K})
$$

which is just the right inequality of (1.3).

According to the equality condition of (3.7), we know that if $\tau \neq \pm 1$, then equality holds in the right inequality of (1.7) if and only if $K$ is centered, i.e., if $K$ is not origin-symmetric, equality holds in the right inequality if and only if $\tau= \pm 1$.

Now, we prove the left inequality of (1.7). Since $f_{1}(0)=f_{2}(0)=\frac{1}{2}$, together with Minkowski's inequality, we have

$$
\begin{aligned}
& B_{p, i}^{(0)}(K)^{\frac{p}{n-i}} \\
& =\left[\frac{1}{n} \int_{S^{n-1}} b_{p}^{(0)}(K, u)^{n-i} d S(u)\right]^{\frac{p}{n-i}} \\
& =\left[\frac{1}{n} \int_{S^{n-1}}\left(\frac{1}{2} h^{p}(K, u)+\frac{1}{2} h^{p}(K,-u)\right)^{\frac{n-i}{p}} d S(u)\right]^{\frac{p}{n-i}} \\
& =\left[\frac{1}{n} \int_{S^{n-1}}\left(\frac{1}{2} f_{1}(\tau) h^{p}(K, u)+\frac{1}{2} f_{2}(\tau) h^{p}(K,-u)+\frac{1}{2} f_{1}(\tau) h^{p}(K,-u)+\frac{1}{2} f_{2}(\tau) h^{p}(K, u)\right)^{\frac{n-i}{p}} d S(u)\right]^{\frac{p}{n-i}} \\
& \leqslant\left[\frac{1}{n} \int_{S^{n-1}}\left(\frac{1}{2} f_{1}(\tau) h^{p}(K, u)+\frac{1}{2} f_{2}(\tau) h^{p}(K,-u)\right)^{\frac{n-i}{p}} d S(u)\right]^{\frac{p}{n-i}}
\end{aligned}
$$




$$
\begin{aligned}
& +\left[\frac{1}{n} \int_{S^{n-1}}\left(\frac{1}{2} f_{1}(\tau) h^{p}(K,-u)+\frac{1}{2} f_{2}(\tau) h^{p}(K, u)\right)^{\frac{n-i}{p}} d S(u)\right]^{\frac{p}{n-i}} \\
= & {\left[\frac{1}{n} \int_{S^{n-1}}\left(f_{1}(\tau) h^{p}(K, u)+f_{2}(\tau) h^{p}(K,-u)\right)^{\frac{n-i}{p}} d S(u)\right]^{\frac{p}{n-i}} } \\
= & {\left[\frac{1}{n} \int_{S^{n-1}} b_{p}^{(\tau)}(K, u)^{n-i} d S(u)\right]^{\frac{p}{n-i}} } \\
= & B_{p, i}^{(\tau)}(K)^{\frac{p}{n-i}} .
\end{aligned}
$$

That is,

$$
\mathrm{B}_{\mathrm{p}, \mathrm{i}}^{(\tau)}(\mathrm{K}) \geqslant \mathrm{B}_{\mathrm{p}, \mathrm{i}}^{(0)}(\mathrm{K})
$$

The equality condition of Minkowski's inequality tells us that if $\tau \neq 0$, then equality holds in the left inequality of (1.7) if and only if $\mathrm{K}$ is centered. Thus if $\mathrm{K}$ is not origin-symmetric, then equality holds in the left inequality of (1.7) if and only if $\tau=0$.

For the case $n-p<i<n$ or $i>n$, the proof is similar.

\section{Brunn-Minkowski type inequalities}

In this section, we establish two Brunn-Minkowski type inequalities for the ith general $\mathrm{L}_{\mathrm{p}}$-mixed width-integral.

Theorem 4.1. If $\tau \in[-1,1], K, L \in \mathcal{K}_{0}^{n}$, and $p>0$, then for $i \leqslant n-p$,

$$
B_{p, i}^{(\tau)}(K+p L)^{\frac{p}{n-i}} \leqslant B_{p, i}^{(\tau)}(K)^{\frac{p}{n-i}}+B_{p, i}^{(\tau)}(L)^{\frac{p}{n-i}},
$$

with equality if and only if $\mathrm{K}$ and $\mathrm{L}$ have similar general $\mathrm{L}_{\mathrm{p}}$-width.

In fact, the more general result than Theorem 4.1 will be obtained as follows.

Theorem 4.2. If $\mathrm{K}, \mathrm{L} \in \mathcal{K}_{\mathrm{o}}^{\mathrm{n}}, \tau \in[-1,1]$, and $\mathrm{p}>0$, then for $\mathrm{i} \leqslant \mathrm{n}-\mathrm{p} \leqslant \mathrm{j} \leqslant \mathrm{n}$ and $\mathrm{i} \neq \mathrm{j}$

$$
\left(\frac{B_{p, i}^{(\tau)}\left(K+_{p} L\right)}{B_{p, j}^{(\tau)}\left(K++_{p} L\right)}\right)^{\frac{p}{j-i}} \leqslant\left(\frac{B_{p, i}^{(\tau)}(K)}{B_{p, j}^{(\tau)}(K)}\right)^{\frac{p}{j-i}}+\left(\frac{B_{p, i}^{(\tau)}(L)}{B_{p, j}^{(\tau)}(L)}\right)^{\frac{p}{j-i}} ;
$$

for $j \geqslant n \geqslant i \geqslant n-p$ and $i \neq j$

$$
\left(\frac{B_{p, i}^{(\tau)}\left(K+_{p} L\right)}{B_{p, j}^{(\tau)}\left(K+_{p} L\right)}\right)^{\frac{p}{j-i}} \geqslant\left(\frac{B_{p, i}^{(\tau)}(K)}{B_{p, j}^{(\tau)}(K)}\right)^{\frac{p}{j-i}}+\left(\frac{B_{p, i}^{(\tau)}(L)}{B_{p, j}^{(\tau)}(L)}\right)^{\frac{p}{j-i}},
$$

with equality in every inequality if and only if $\mathrm{K}$ and $\mathrm{L}$ have similar general $\mathrm{L}_{\mathrm{p}}$-width.

To complete the proof of Theorem 4.2, the following lemmas may be required. An extension of Beckenbach's inequality (see [3]) was obtained by Dresher (see [5]) through the means of moment-space techniques.

Lemma 4.3 (Beckenbach-Dresher inequality). If $p \geqslant 1 \geqslant r \geqslant 0, p \neq r, f, g \geqslant 0$, and $\phi$ is a distribution function, then

$$
\left(\frac{\int_{\mathbb{E}}(f+g)^{p} d \phi}{\int_{\mathbb{E}}(f+g)^{r} d \phi}\right)^{\frac{1}{p-r}} \leqslant\left(\frac{\int_{\mathbb{E}} f^{p} d \phi}{\int_{\mathbb{E}} f^{r} d \phi}\right)^{\frac{1}{p-r}}+\left(\frac{\int_{\mathbb{E}} g^{p} d \phi}{\int_{\mathbb{E}} g^{r} d \phi}\right)^{\frac{1}{p-r}},
$$

with equality if and only if the functions $\mathrm{f}$ and $\mathrm{g}$ are positively proportional.

Here $\mathbb{E}$ is a bounded measurable subset in $\mathbb{R}^{n}$.

The inverse Beckenbach-Dresher inequality was established in the reference [18]. 
Lemma 4.4 (Inverse Beckenbach-Dresher inequality). If $1 \geqslant p \geqslant 0 \geqslant r, p \neq r, f, g \geqslant 0$, and $\phi$ is a distribution function, then

$$
\left(\frac{\int_{\mathbb{E}}(f+g)^{p} d \phi}{\int_{\mathbb{E}}(f+g)^{r} d \phi}\right)^{\frac{1}{p-r}} \geqslant\left(\frac{\int_{\mathbb{E}} f^{p} d \phi}{\int_{\mathbb{E}} f^{r} d \phi}\right)^{\frac{1}{p-r}}+\left(\frac{\int_{\mathbb{E}} g^{p} d \phi}{\int_{\mathbb{E}} g^{r} d \phi}\right)^{\frac{1}{p-r}},
$$

with equality if and only if the functions $\mathrm{f}$ and $\mathrm{g}$ are positively proportional.

Proof of Theorem 4.2. From (1.3) and (4.3), it follows that for $i \leqslant n-p \leqslant j \leqslant n$

$$
\begin{aligned}
& \left(\frac{B_{p, i}^{(\tau)}\left(K+_{p} L\right)}{B_{p, j}^{(\tau)}(K+p L)}\right)^{\frac{p}{j-i}} \\
& =\left(\frac{\frac{1}{n} \int_{S^{n-1}} b_{p}^{(\tau)}\left(K+_{p} L, u\right)^{n-i} d S(u)}{\frac{1}{n} \int_{S^{n-1}} b_{p}^{(\tau)}(K+p L, u)^{n-j} d S(u)}\right)^{\frac{p}{j-i}}
\end{aligned}
$$

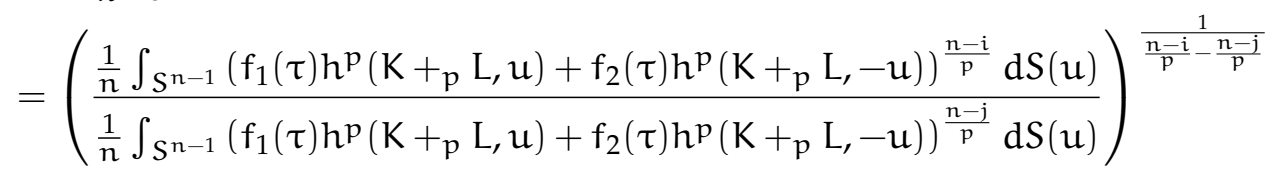

$$
\begin{aligned}
& =\left(\frac{\frac{1}{n} \int_{S^{n-1}}\left(f_{1}(\tau) h^{p}(K, u)+f_{2}(\tau) h^{p}(K,-u)+f_{1}(\tau) h^{p}(L, u)+f_{2}(\tau) h^{p}(L,-u)\right)^{\frac{n-i}{p}} d S(u)}{\frac{1}{n} \int_{S^{n-1}}\left(f_{1}(\tau) h^{p}(K, u)+f_{2}(\tau) h^{p}(K,-u)+f_{1}(\tau) h^{p}(L, u)+f_{2}(\tau) h^{p}(L,-u)\right)^{\frac{n-j}{p}} d S(u)}\right)^{\frac{1}{p-i}-\frac{n-j}{p}} \\
& \leqslant\left(\frac{\frac{1}{n} \int_{S^{n-1}}\left(f_{1}(\tau) h^{p}(K, u)+f_{2}(\tau) h^{p}(K,-u)\right)^{\frac{n-i}{p}} d S(u)}{\frac{1}{n} \int_{S^{n-1}}\left(f_{1}(\tau) h^{p}(K, u)+f_{2}(\tau) h^{p}(K,-u)\right)^{\frac{n-j}{p}} d S(u)}\right)^{\frac{p}{j-i}} \\
& +\left(\frac{\frac{1}{n} \int_{S^{n-1}}\left(f_{1}(\tau) h^{p}(L, u)+f_{2}(\tau) h^{p}(L,-u)\right)^{\frac{n-i}{p}} d S(u)}{\frac{1}{n} \int_{S^{n-1}}\left(f_{1}(\tau) h^{p}(L, u)+f_{2}(\tau) h^{p}(L,-u)\right)^{\frac{n-j}{p}} d S(u)}\right)^{\frac{p}{j-i}} \\
& =\left(\frac{B_{p, i}^{(\tau)}(K)}{B_{p, j}^{(\tau)}(K)}\right)^{\frac{p}{j-i}}+\left(\frac{B_{p, i}^{(\tau)}(L)}{B_{p, j}^{(\tau)}(L)}\right)^{\frac{p}{j-i}} \text {. }
\end{aligned}
$$

This gets the desired inequality (4.1). Using the same method, inequality (4.2) follows from inequality (4.4).

Together with the equality conditions of inequalities (4.3) and (4.4), we see that equality holds in inequalities (4.1) and (4.2) if and only if $b_{p}^{(\tau)}(K, u)$ and $b_{p}^{(\tau)}(L, u)$ are positively proportional, namely, $K$ and $\mathrm{L}$ have similar general $\mathrm{L}_{\mathrm{p}}$-width.

Let $j=n$ in (4.1). Since $B_{p, n}^{(\tau)}(K+L)=B_{p, n}^{(\tau)}(K)=B_{p, n}^{(\tau)}(L)=\omega_{n}$ is a constant, we obtain Theorem 4.1.

\section{Acknowledgment}

This research is supported by the Natural Science Foundation of China (Grant No. 11371239).

\section{References}

[1] J. Abardia, A. Bernig, Projection bodies in complex vector spaces, Adv. Math., 227 (2011), 830-846. 1

[2] S. Alesker, A. Bernig, F. E. Schuster, Harmonic analysis of translation invariant valuations, Geom. Funct. Anal., 21 (2011), 751-773. 1

[3] E. F. Beckenbach, R. Bellman, Inequalities, Second revised printing, Ergebnisse der Mathematik und ihrer Grenzgebiete. Neue Folge, Band 30 Springer-Verlag, New York, Inc., (1965). 4 
[4] W. Blaschke, Vorlesungen über Integralgeometrie, (German) 3te Aufl, Deutscher Verlag der Wissenschaften, Berlin, (1955). 1

[5] M. Dresher, Moment spaces and inequalities, Duke Math. J., 20 (1953), 261-271. 4

[6] Y.-B. Feng, General mixed width-integral of convex bodies, J. Nonlinear Sci. Appl., 9 (2016), 4226-4234. 1

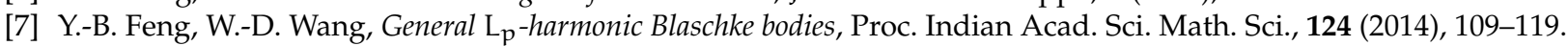
1

[8] Y.-B. Feng, W. Weidong, L. Fenghong, Some inequalities on general $\mathrm{L}_{\mathrm{p}}$-centroid bodies, Math. Inequal. Appl., 18 (2015), 39-49. 1

[9] R. J. Gardner, Geometric tomography, Second edition, Encyclopedia of Mathematics and its Applications, Cambridge University Press, New York, (2006). 1, 2, 2

[10] C. Haberl, Lp intersection bodies, Adv. Math., 217 (2008), 2599-2624. 1

[11] C. Haberl, Minkowski valuations intertwining with the special linear group, J. Eur. Math. Soc. (JEMS), 14 (2012), 15651597.

[12] C. Haberl, M. Ludwig, A characterization of $\mathrm{L}_{p}$ intersection bodies, Int. Math. Res. Not., 2006 (2006), 29 pages.

[13] C. Haberl, F. E. Schuster, Asymmetric affine $\mathrm{L}_{\mathrm{p}}$ Sobolev inequalities, J. Funct. Anal., 257 (2009), 641-658.

[14] C. Haberl, F. E. Schuster, General $\mathrm{L}_{p}$ affine isoperimetric inequalities, J. Differential Geom., 83 (2009), 1-26.

[15] C. Haberl, F. E. Schuster, J. Xiao, An asymmetric affine Pólya-Szegö principle, Math. Ann., 352 (2012), 517-542. 1

[16] G. H. Hardy, J. E. Littlewood, G. Pólya, Pu teng shih, (Chinese) [[Inequalities]] Translated from the English by Yüeh Min-yi Science Press, Peking, (1965). 1, 3, 3, 3, 3, 3

[17] G.-S. Leng, C.-J. Zhao, B.-W. He, X.-Y. Li, Inequalities for polars of mixed projection bodies, Sci. China Ser. A, 47 (2004), 175-186. 1

[18] X.-Y. Li, C.-J. Zhao, On the p-mixed affine surface area, Math. Inequal. Appl., 17 (2014), 443-450. 4

[19] M. Ludwig, Valuations in the affine geometry of convex bodies, Integral geometry and convexity, World Sci. Publ., Hackensack, NJ, (2004), 49-65. 1

[20] M. Ludwig, Minkowski valuations, Trans. Amer. Math. Soc., 357 (2005), 4191-4213. 1

[21] E. Lutwak, A general Bieberbach inequality, Math. Proc. Cambridge Philos. Soc., 78 (1975), 493-495. 1

[22] E. Lutwak, Dual mixed volumes, Pacific J. Math., 58 (1975), 531-538. 1, 3.1

[23] E. Lutwak, Mixed width-integrals of convex bodies, Israel J. Math., 28 (1977), 249-253. 1

[24] E. Lutwak, Extended affine surface area, Adv. Math., 85 (1991), 39-68. 2

[25] E. Lutwak, Inequalities for mixed projection bodies, Trans. Amer. Math. Soc., 339 (1993), 901-916. 1

[26] E. Lutwak, The Brunn-Minkowski-Firey theory, I, Mixed volumes and the Minkowski problem, J. Differential Geom., 38 (1993), 131-150. 2

[27] E. Lutwak, D. Yang, G.-Y. Zhang, $\mathrm{L}_{\mathrm{p}}$-affine isoperimetric inequalities, J. Differential Geom., 56 (2000), 111-132. 1

[28] E. Lutwak, D. Yang, G.-Y. Zhang, Orlicz centroid bodies, J. Differential Geom., 84 (2010), 365-387.

[29] E. Lutwak, D. Yang, G.-Y. Zhang, Orlicz projection bodies, Adv. Math., 223 (2010), 220-242. 1

[30] R. Schneider, Convex bodies: the Brunn-Minkowski theory, Second expanded edition, Encyclopedia of Mathematics and its Applications, Cambridge University Press, Cambridge, (2014). 1, 2, 2

[31] F. E. Schuster, Convolutions and multiplier transformations of convex bodies, Trans. Amer. Math. Soc., 359 (2007), 5567-5591. 1

[32] F. E. Schuster, Crofton measures and Minkowski valuations, Duke Math. J., 154 (2010), 1-30.

[33] W.-D. Wang, Y.-B. Feng, A general $\mathrm{L}_{\mathrm{p}}$-version of Petty's affine projection inequality, Taiwanese J. Math., 17 (2013), 517-528.

[34] W.-D. Wang, T.-Y. Ma, Asymmetric $\mathrm{L}_{\mathrm{p}}$-difference bodies, Proc. Amer. Math. Soc., 142 (2014), 2517-2527.

[35] W. Weidong, W. Xiaoyan, Shephard type problems for general $\mathrm{L}_{\mathrm{p}}$-projection bodies, Taiwanese J. Math., 16 (2012), 1749-1762. 1 